Cite this: Phys. Chem. Chem. Phys., 2011, 13, 12447-12453

\title{
Trends in water monomer adsorption and dissociation on flat insulating surfaces
}

\author{
Xiao Liang Hu, ${ }^{a}$ Javier Carrasco, ${ }^{a b}$ Jiří Klimeš $^{a}$ and Angelos Michaelides*a \\ Received 21st March 2011, Accepted 13th May 2011 \\ DOI: $10.1039 / \mathrm{c} 1 \mathrm{cp20846b}$
}

The interaction of water with solid surfaces is key to a wide variety of industrial and natural processes. However, the basic principles that dictate how stable and in which state (intact or dissociated) water will be on a given surface are not fully understood. Towards this end, we have used density functional theory to examine water monomer adsorption on the (001) surfaces of a broad range of alkaline earth oxides, alkaline earth sulfides, alkali fluorides, and alkali chlorides. Some interesting general conclusions are arrived at: (i) on all the surfaces considered only a few specific adsorption structures are favoured; (ii) water becomes more stable upon descending the oxide and fluoride series but does not vary much upon going down the chloride and sulfide series; (iii) water is stabilised both by an increase in the lattice constant, which facilitates hydrogen bonding to the substrate, and by the flexibility of the substrate. These are also factors that favour water dissociation. We hope that this study is of some value in better understanding the surface science of water in general, and in assisting in the interpretation and design of future experiments.

\section{Introduction}

The interaction of water with metal, oxide, and semiconductor surfaces is important to a wide variety of scientific disciplines and industrial applications. ${ }^{1-6}$ When seeking molecular-level understanding, the fundamental issues that arise for water at interfaces generally concern establishing the adsorption structures and stability of water and in determining if the molecules dissociate or remain intact. The properties of interfacial water depend sensitively on the answers to these questions. For example, water dissociation at metal surfaces can be the first step in oxidation leading to corrosion or at oxide surfaces can lead to oxidation of water to generate hydrogen and oxygen. ${ }^{1-10}$ Similarly water dissociation on certain oxide surfaces can lead to rapid proton transfer and may have implications for proton conducting electrolytes. ${ }^{12}$

Many studies have examined the interaction of water with various solid surfaces. Two encyclopaedic reviews demonstrate the enormous body of work done within a surface science perspective alone, i.e. in the low temperature and generally low coverage regime. ${ }^{1,3}$ Despite all this work, general insight of a predictive nature is limited and trends are yet to be established to predict how water will adsorb on a given unstudied surface. This is in marked contrast to other areas of surface chemistry, e.g., in heterogeneous catalysis, where

${ }^{a}$ London Centre for Nanotechnology and Department of Chemistry, University College London, London WC1E 6BT, UK.

E-mail: angelos.michaelides@ucl.ac.uk

${ }^{b}$ Fritz-Haber-Institut der Max-Planck-Gesellschaft, Faradayweg 4-6, 14195 Berlin, Germany clear correlations have been identified and exploited (see e.g. ref. 13-15). Water on metals is probably the specific area where there is the clearest understanding at present. It is, for example, known that water monomers tend to adsorb above individual metal atoms of the substrate with the molecular plane almost parallel to the surface. ${ }^{16}$ The tendency to dissociate increases upon going from right to left across the transition metal series. Likewise water molecules incorporated in hydrogen bonded adlayers or adsorbed at defect sites exhibit an increased tendency to dissociate. ${ }^{17-19}$ For water on other surfaces such as oxides, salts, or minerals similar such trends are less well understood. Indeed detailed understanding of water adsorption on oxide and mineral surfaces is often obtained on a case-by-case basis. For example, water on $\mathrm{MgO}$ and $\mathrm{TiO}_{2}$ has been widely and, at times, controversially discussed. ${ }^{3,19-28}$ Likewise water on salts has been the focus of much attention, mainly with a view to better understanding salt dissolution and caking. ${ }^{5,29-31}$

Here we report a systematic density functional theory (DFT) study of water monomer adsorption on a range of surfaces. So as to identify the inherent reactivity of each surface we focus exclusively on water monomer adsorption and dissociation on a series of flat (001) surfaces. The following materials - all with the rocksalt structure-were examined: alkaline earth oxides $(\mathrm{MgO}, \mathrm{CaO}, \mathrm{SrO}$, and $\mathrm{BaO})$; alkaline earth sulfides (MgS, CaS, $\mathrm{SrS}$, and $\mathrm{BaS})$; alkali fluorides ( $\mathrm{LiF}$, $\mathrm{NaF}, \mathrm{KF}, \mathrm{RbF}$, and $\mathrm{CsF})$; and alkali chlorides $(\mathrm{LiCl}, \mathrm{NaCl}$, $\mathrm{KCl}$, and $\mathrm{RbCl}$ ). Most of these compounds are of practical interest. For example, alkaline earth oxides have been studied extensively as simple models of ionic materials or as thin film 
substrates in surface science. ${ }^{32-34}$ Alkali fluorides and chlorides are related to our daily life, for example, $\mathrm{NaCl}$ is studied to understand salt dissolution, ${ }^{29-31,35}$ and $\mathrm{NaF}$ is used in toothpaste. The main results to come from this study are: (i) despite examining a wide range of surfaces only three to four specific adsorption structures are favoured; (ii) the stability of the water monomer increases upon descending the oxide and fluoride series but remains rather constant upon going down the chloride and sulfide series; (iii) water is stabilised both by an increase in the lattice constant - which facilitates hydrogen bonding with the substrate - and by the flexibility of the substrate. These are also factors that facilitate water dissociation.

The remainder of this article is organised as follows. Computational details are briefly presented in the next section. In Section III adsorption structures and the general trends observed with regard to water adsorption and dissociation are discussed. Following this, factors affecting water adsorption and dissociation are analysed. Finally, Section IV summarizes our main conclusions.

\section{Computational details}

DFT calculations were performed with the periodic plane wave code $\mathrm{VASP}^{36,37}$ using the $\mathrm{PBE}^{38}$ exchange-correlation functional, PAW potentials, ${ }^{39-41}$ and a $400 \mathrm{eV}$ plane wave cutoff. All calculations involved (001) surfaces in which the slabs were three layers thick with a fixed bottom layer in a $(1.5 \times 1.5) \sqrt{ } 2 a_{0}$ surface cell. An eight layer thick slab yields an adsorption energy of (intact or dissociated) water on each surface within $10 \mathrm{meV}$ of that obtained with the three layer thick slab. ${ }^{42}$ A $2 \times 2 \times 1$ Monkhorst- Pack $^{43} k$ point mesh was used to sample the Brillouin zone. The vacuum region between slabs is $15 \AA$ and the dipole across the slab, present because of adsorption on just one side of the slab, was corrected for. ${ }^{44}$ During structure optimizations all forces on relaxed ions are reduced below $15 \mathrm{meV}^{-1}$. It has been observed that the PBE values of the bulk lattice constant $\left(a_{0}\right)$ are slightly overestimated compared to experimental values. ${ }^{45}$ Our values are similar to previous PBE or PW91 calculations, in particular for oxides. ${ }^{46-48}$ Tests to establish if van der Waals ( $\mathrm{vdW}$ ) dispersion forces alter any of the conclusions drawn have been performed with a modified version of the non-local vdW density functional of Dion et al., ${ }^{49}$ referred to as "optB88-vdW". ${ }^{50}$ The numerical set-up for optB88-vdW calculations is the same as for PBE, except that a $600 \mathrm{eV}$ plane wave cutoff was used. As we briefly discuss later, accounting for $\mathrm{vdW}$ dispersion forces does not change the adsorption structures or trends obtained from PBE.

Some quantities used in this article are defined here. The adsorption energy, $E_{\mathrm{ads}}$, of a water monomer on the (001) surface is defined by

$$
E_{\mathrm{ads}}=E_{\mathrm{water} / \mathrm{MN}}-E_{\mathrm{MN}}-E_{\mathrm{water}},
$$

where $E_{\mathrm{water} / \mathrm{MN}}, E_{\mathrm{MN}}$, and $E_{\mathrm{water}}$ are the total energies of (the intact or dissociated) water monomers adsorbed on the (001) surface, the clean surface, and the isolated water molecule, respectively. In the analysis below, the adsorption energy is decomposed into energy contributions from the energy gain due to bonding to the surface and the energy cost associated with deforming the water and substrate upon creation of the adsorption complex. The part designed to reveal exclusively the interaction between the water monomer and the substrate, $E_{\text {bond, }}$, is defined as

$$
E_{\mathrm{bond}}=E_{\mathrm{water} / \mathrm{MN}}-E_{\mathrm{MN}}^{*}-E_{\mathrm{water}}^{*},
$$

where $E_{\mathrm{MN}}^{*}$ and $E_{\mathrm{water}}^{*}$ are the total energies of the isolated clean slab and the isolated water monomer in vacuum, each fixed in the structure they assume in the adsorption system. The energetic contribution coming from structural deformation of the water monomer and substrate, $E_{\text {str }}$, is defined as

$$
E_{\mathrm{str}}=E_{\mathrm{MN}}^{*}+E_{\mathrm{water}}^{*}-E_{\mathrm{MN}}-E_{\mathrm{water}}
$$

where all terms are defined as in eqn (1) and (2). Finally, the degree of rumpling of the (001) surfaces is evaluated by

$$
\Delta d=d_{\mathrm{M}}-d_{\mathrm{N}},
$$

where $d_{\mathrm{M}}$ and $d_{\mathrm{N}}$ are the heights of the metal (cation) and nonmetal (anion) ions of the outermost $\mathrm{MN}(\mathrm{M}=\mathrm{Mg}, \mathrm{Ca}, \mathrm{Sr}$, $\mathrm{Ba}, \mathrm{Li}, \mathrm{Na}, \mathrm{K}, \mathrm{Rb}, \mathrm{Cs}$ and $\mathrm{N}=\mathrm{O}, \mathrm{S}, \mathrm{F}, \mathrm{Cl}$ ) layer. With this definition a positive $\Delta d$ indicates that the metal ions (M) in the outermost layer relax upwards (i.e., further out into the vacuum) with respect to the non-metal ions $(\mathrm{N})$.

\section{Adsorption and dissociation trends}

\section{A Adsorption structure and energy}

First we discuss the main adsorption structures examined for intact and dissociated water monomers. Despite considering a large variety of initial adsorption structures, ${ }^{51}$ interestingly only four basic types of structures have been identified. These are shown in Fig. 1 and labelled as Type I to IV. In the Type I structure, the oxygen of the water monomer is above or close to a metal ion of the substrate with the plane of the molecule almost parallel to the surface or inclined slightly asymmetrically with respect to the surface normal. The interaction with the substrate is mainly electrostatic in nature between oxygen and the cation beneath it. This is the preferred adsorption structure for intact water on $\mathrm{MgO}, \mathrm{MgS}, \mathrm{CaS}, \mathrm{LiF}, \mathrm{LiCl}$, and $\mathrm{NaCl}$. In the Type II adsorption structure the oxygen of the water molecule is located on a hollow site and the $\mathrm{OH}$ bonds are

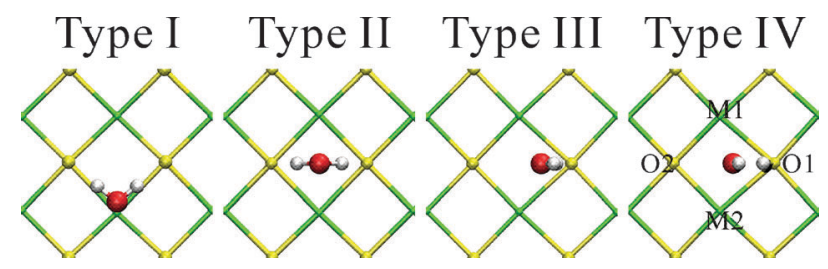

Fig. 1 Adsorption structure of the most stable (intact and dissociated) water monomer on $\mathrm{MN}(\mathrm{M}=\mathrm{Mg}, \mathrm{Ca}, \mathrm{Sr}, \mathrm{Ba}, \mathrm{Li}, \mathrm{Na}, \mathrm{K}, \mathrm{Rb}, \mathrm{Cs}$, and $\mathrm{N}=\mathrm{O}, \mathrm{S}, \mathrm{F}, \mathrm{Cl})(001)$ surfaces. The Type I adsorption structure is found on $\mathrm{MgO}, \mathrm{MgS}, \mathrm{CaS}, \mathrm{LiF}, \mathrm{LiCl}$, and $\mathrm{NaCl}$; Type II on $\mathrm{CaO}, \mathrm{SrO}, \mathrm{BaO}$, $\mathrm{SrS}$, BaS, NaF, KF, RbF, KCl, and $\mathrm{RbCl}$; Type III on $\mathrm{CsF}$ and $\mathrm{RbF}$. Dissociated Type IV water structure is found on $\mathrm{CaO}, \mathrm{SrO}$, and $\mathrm{BaO}$. Note that the most stable adsorption structure on some surfaces is not as symmetric as the idealised structures shown here. In the Type IV structures, some ions are labelled for discussion purposes in the text. White, red (dark), yellow (grey), and green (light) spheres represent water hydrogen, water oxygen, surface non-metal ions, and surface metal ions, respectively. 
directed towards the surface anion sites. This adsorption structure is mediated by hydrogen bonding with the two substrate anions to which the two $\mathrm{OH}$ bonds are directed. This is the preferred adsorption structure for intact water on $\mathrm{CaO}, \mathrm{SrO}, \mathrm{BaO}, \mathrm{SrS}, \mathrm{BaS}, \mathrm{NaF}, \mathrm{KF}, \mathrm{RbF}, \mathrm{KCl}$, and $\mathrm{RbCl}$. In the Type III structure the water molecule is located on the hollow site, with only one $\mathrm{OH}$ bond directed toward the surface non-metal sites. This structure is again mediated by hydrogen bonds and it is the preferred adsorption structure for intact water on $\mathrm{CsF}$ and equally stable for water on $\mathrm{RbF}$ as the Type II structure. In the first three types of structures, the water molecule is intact. In contrast, the Type IV structure is the most stable structure of the dissociated water monomer on $\mathrm{CaO}, \mathrm{SrO}$, and $\mathrm{BaO}$. It involves an adsorbed hydrogen on a surface oxygen site and a hydroxyl above the adjacent metal ion with the $\mathrm{OH}$ bond directed toward the vacuum.

The adsorption energies for the most stable (intact and dissociated) water monomer on each surface are given in Fig. 2, plotted as a function of the lattice constant. The values range from about -0.2 to $-1.6 \mathrm{eV}$. Where comparison with previous $\mathrm{PBE}$ adsorption energies on flat rocksalt-like surfaces is possible, the agreement is good. ${ }^{19,29,46,52,53}$ Fig. 2 also illustrates several important features of water adsorption on the various surfaces. First, the adsorption energy is rather constant on the sulfide and chloride series, whilst it increases when descending the oxide and fluoride series. Second, the water monomer remains intact on the sulfide, fluoride, and chloride surfaces, but dissociates on the oxide surfaces except for $\mathrm{MgO}$. Third, the dissociated adsorption structures on most of the oxide surfaces have larger adsorption energies $(>-0.9 \mathrm{eV})$ than the intact adsorption structures on all examined surfaces. One further periodic trend identified is that we find that the preferred structure for the monomer changes from Type I to Type II or Type III as one descends each periodic series.

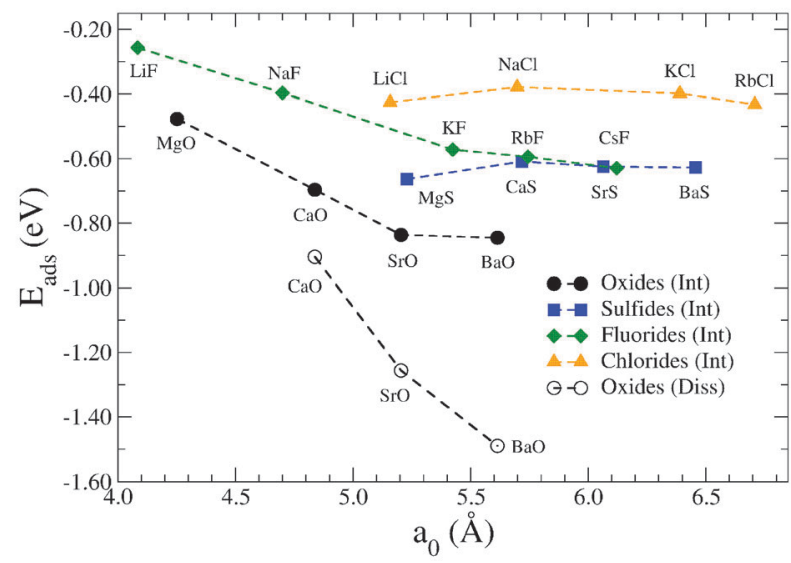

Fig. 2 Adsorption energies of the most stable (intact and dissociated) monomer structures on $\mathrm{MN}$ (001) surfaces as a function of the lattice constant. Filled symbols and unfilled symbols indicate the adsorption energies of the most stable intact (labelled "Int") and dissociated (labelled "Diss") monomer structures, respectively. Stable intact monomer structures (with a negative adsorption energy) were identified on all examined surfaces. However, stable dissociated monomer structures were identified only on $\mathrm{CaO}, \mathrm{SrO}$, and $\mathrm{BaO}$.
The adsorption energies reported herein have been obtained with the PBE exchange-correlation functional. Although this functional is widely used in water adsorption studies it does not account for vdW dispersion forces. Tests with the non-local "optB88-vdW",50 functional yield adsorption energies on the oxide and chloride series about 0.10 to 0.15 $\mathrm{eV}$ more favourable than those obtained with PBE, which is consistent with recent results for water-ice on metals. ${ }^{54}$ However, on each of the substrates examined, none of the basic adsorption structures obtained with PBE differ when the optB88-vdW functional is used and none of the key trends observed are altered.

The four classes of adsorption structures and the various periodic trends are the key results of this study. We now consider the adsorption systems in more detail in order to understand why these trends emerge.

\section{B Intact water monomer analysis}

We begin the analysis by discussing the adsorption of the intact water monomers. As we have seen almost all monomer structures are either of Type I or Type II and so it is interesting to consider the factors that control the stabilities of these two structures. To investigate this in as "clean" a manner as possible, we examined an idealised Type I structure (with the water oxygen fixed above a surface cation) and an idealised Type II structure (with the monomer on a hollow site forming two hydrogen bonds with the surface anions). ${ }^{55}$ The adsorption energies of these two structures on the alkaline earth oxide and sulfide series are plotted in Fig. 3 as a function of the lattice constant. As the substrate is altered, quite different behaviour is observed for the two structures. Whereas the Type I structure becomes less stable upon increasing the lattice constant in each series, the Type II structure becomes more stable (Fig. 3).

The contrasting behaviour of the two classes of adsorption structures is a result of their different interaction modes with the surface. In the Type I structure the main interaction with the surface is between the metal ion and the oxygen of the water molecule. Careful analysis of the electronic properties of the Type I adsorption systems - involving consideration of the

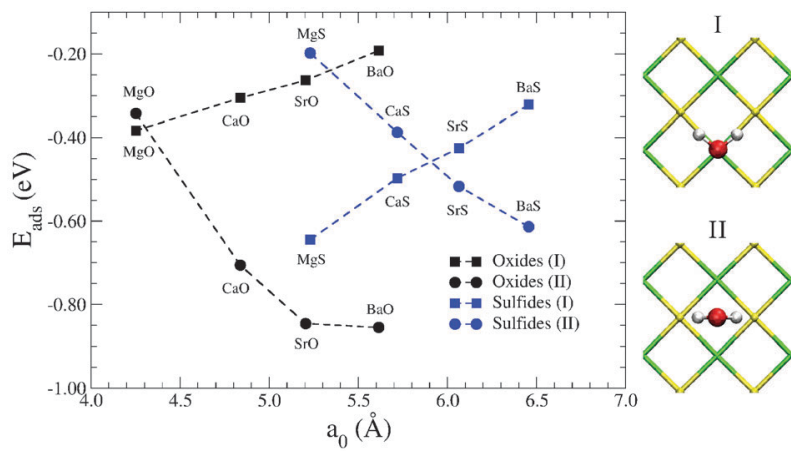

Fig. 3 Adsorption energies of the idealised Type I and II water monomer structures on oxide and sulfide (001) surfaces as a function of the lattice constant. In the idealised Type I structure the water oxygen is fixed above a surface metal ion and in the idealised Type II structure the water monomer is fixed symmetrically above a hollow site between two metal and two non-metal ions. 


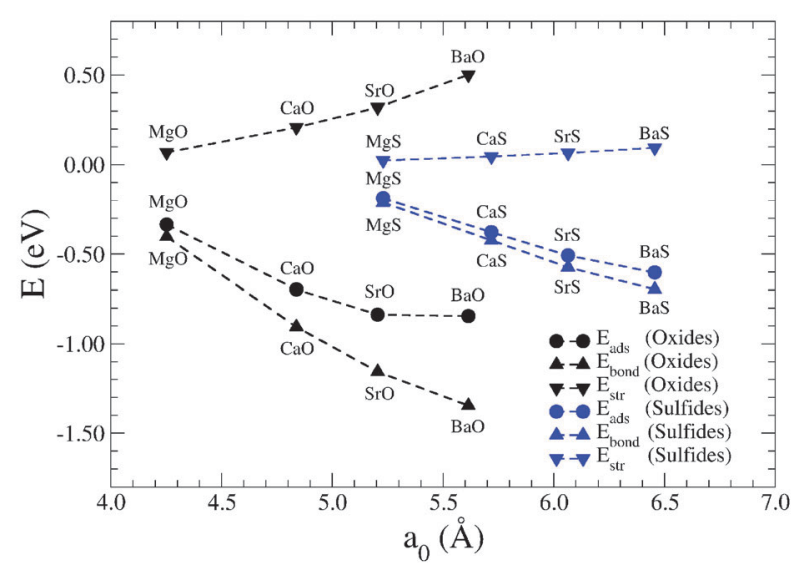

Fig. 4 Adsorption energies and their decomposition for the Type II monomer structure on oxide and sulfide (001) surfaces as a function of the lattice constant. $E_{\mathrm{ads}}, E_{\mathrm{bond}}$, and $E_{\mathrm{str}}$ are defined in eqn (1)-(3), respectively.

partial density of states (PDOS) of individual ions, the occupied Kohn-Sham orbitals, and electron density rearrangement plots - shows that the interaction with the substrate is mainly electrostatic (see ref. 56 for more details). Therefore, with the increase in size of the metal ion, the electrostatic interaction with the surface is reduced and the water-metal ion interaction is weakened. This is reflected, for example, by about a $0.8 \AA$ increase in the height of the water oxygen above the surface upon descending the oxide and sulfide series.

In contrast in the Type II structure the water monomer forms hydrogen bonds with surface anions and adsorption becomes more favourable upon descending the series. To better understand this we decomposed the adsorption energies into: (i) the interaction energy between the deformed water and the deformed substrate, $E_{\text {bond }}($ eqn (2)); and (ii) the energy cost to deform the water and the substrate to the adsorption structure, $E_{\text {str }}$ (eqn (3)). As shown in Fig. $4,{ }^{57}$ the interaction energy between water and the surface increases from the top to the bottom of the series, i.e. it follows the same trend as the adsorption energy. In contrast, the structural deformation part becomes more positive (unfavourable). This more costly deformation energy and the more negative interaction energy are in agreement with the structures obtained. The $\mathrm{OH}$ bond lengths and the internal bond angles of the monomers increase by up to $0.06 \AA$ and $6^{\circ}$ upon descending each series. Likewise the height of the water molecule above the surface decreases by about 22 to $26 \%$ upon descending each series and the length of the hydrogen bonds between water and the surface decreases by about 9 to $13 \%$. The implication of shorter hydrogen bonds is, of course, that they are stronger. Thus, considering the electrostatic nature of hydrogen bonds on these surfaces, ${ }^{56}$ it is the ability of the molecule to form stronger hydrogen bonds that is key to the increased adsorption energy as one moves down each series. The strengthening of hydrogen bond(s) is ultimately related to geometric effects: the increasing lattice constant (i.e., the increasing space between non-metal ions) as one moves down each series enables the molecule to approach the surface more closely and form stronger hydrogen bonds with it.
It is clear that the most stable intact water monomer structure on each surface is determined by the relative strengths of the hydrogen bonds and the oxygen-metal ion interaction. An interesting implication of this is that the switch from the intact Type I to the intact Type II structure occurs in principle further down the sulfide series (between $\mathrm{CaS}$ and $\mathrm{SrS}$ ) than on the oxide series (between $\mathrm{MgO}$ and $\mathrm{CaO}$ ). This arises because the hydrogen bonds to the oxide surfaces are stronger than those on the sulfide surfaces (as indicated by $E_{\text {bond }}$ in Fig. 4), whereas the water-cation interaction is stronger on the sulfides than on the oxides as indicated by the about $0.1 \AA$ longer water oxygen-metal distances on the oxides than on the sulfides.

The idealised Type I and II structures have also been examined on the fluoride and chloride series (not shown). Similar conclusions can be drawn and the behaviour on the fluoride series is like that on the oxide series, whereas the behaviour on the chloride series mimics that on the sulfides. Specifically, the adsorption energies are rather constant on the sulfide and chloride series and become more negative on the oxide and fluoride series.

\section{Dissociated monomer analysis}

We now discuss the dissociated water adsorption structure (i.e. the Type IV structure), which is stable only on $\mathrm{CaO}, \mathrm{SrO}$, and $\mathrm{BaO}$. In particular we explore the role of the substrate in dictating the stability of the dissociated state, focussing on both the flexibility and the lattice constant of the substrate.

Let us first consider the role of surface flexibility, i.e., surface rumpling $(\Delta d$, eqn (4)). This is particularly important for the dissociated state because the substrate atoms beneath the adsorption complex are drawn out of the surface in the presence of the $\mathrm{OH}$ and $\mathrm{H}$ adsorbates. In particular the metal ions beneath the adsorbed hydroxyl can move upwards by as much as about $0.2 \AA$. This displacement of the substrate atoms upon adsorption is a key aspect of the stability of the dissociated state as revealed by a complementary set of calculations on a fixed substrate. Specifically in Table 1 we report the adsorption energies on the relaxed and unrelaxed oxide surfaces. In each case the dissociated state is about $0.4 \mathrm{eV}$ less stable on the unrelaxed substrate compared to the relaxed one. On $\mathrm{CaO}$ this is actually sufficient to make the intact state more stable than the dissociated state when the surface is fixed. Upon inspection of the (vertical) displacement of the surface ions close to the dissociated monomer on the

Table 1 Water adsorption energy on relaxed and unrelaxed (001) oxide surfaces with the Type IV (dissociated) structure. On the relaxed surfaces only the ions in the bottom layer of the slab are fixed and the other atoms including water are fully relaxed. On the unrelaxed surfaces all substrate ions are artificially fixed at the fully relaxed clean surface positions while water is relaxed. The text in the parentheses indicates whether the water monomer is dissociated or intact in each case. All values are in $\mathrm{eV}$.

\begin{tabular}{lll}
\hline$E_{\text {ads }}$ & Relaxed & Unrelaxed \\
\hline $\mathrm{MgO}$ & -0.34 (Int) & -0.30 (Int) \\
$\mathrm{CaO}$ & -0.90 (Diss) & -0.61 (Int) \\
$\mathrm{SrO}$ & -1.26 (Diss) & -0.87 (Diss) \\
$\mathrm{BaO}$ & -1.49 (Diss) & -1.11 (Diss) \\
\hline
\end{tabular}




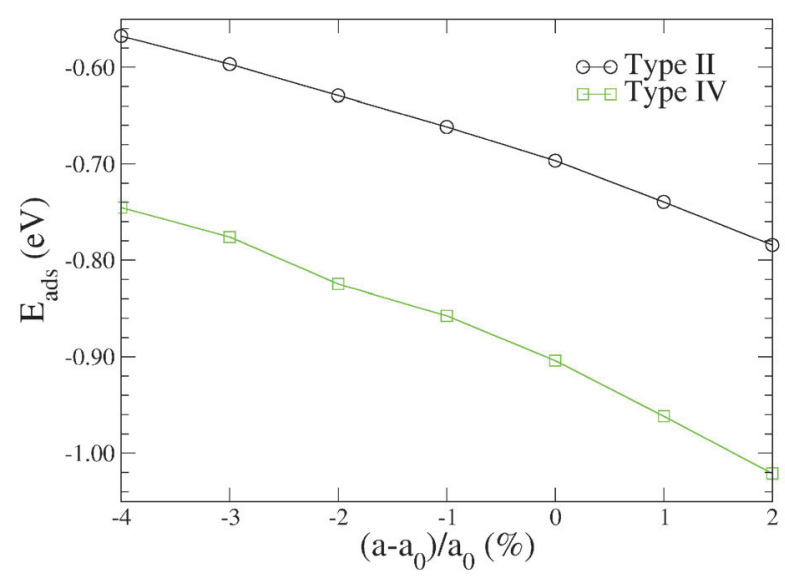

Fig. 5 Adsorption energies of water on $\mathrm{CaO}$ as a function of the percentage deviation of the lattice constant from the PBE-optimized lattice constant $\left(a_{0}\right)$ of $4.84 \AA$. Results for Type II and Type IV structures are shown.

relaxed surfaces, we see that the metal ions (M1 and $\mathrm{M} 2$ in Fig. 1) have moved upwards about $0.2 \AA$ for $\mathrm{CaO}, \mathrm{SrO}$, and $\mathrm{BaO}$, and only $0.04 \AA$ for $\mathrm{MgO}$. In contrast, the upward (or downward) displacement of the oxygen ions ( $\mathrm{O} 1$ and $\mathrm{O} 2$ in Fig. 1) is smaller than about $0.1 \AA$. This leads to a larger (local) surface rumpling than that on the clean surfaces. ${ }^{58}$

We now consider the role of the lattice constant (or the strain in the surface plane). At transition metal surfaces, surface strain can affect surface reactivity through shifts of the $d$-band centre (see e.g. ref. 59). The electronic properties of flat ionic (oxide, sulfide, fluoride, and chloride) surfaces differ, of course, from those of metal surfaces yet a similar correlation between reactivity (adsorption energy) and the substrate lattice strain is observed for the ionic substrates considered here. As shown in Fig. 5, for the example of $\mathrm{CaO}$, there is a clear correlation between the lattice constant and adsorption energy (for both the intact Type II and dissociated Type IV structures). Specifically, as the lattice constant is increased from $-4 \%$ to $+2 \%$ of the equilibrium value, the adsorption energy increases steadily. Expanding upon this analysis, we exchanged the lattice constants of $\mathrm{CaO}$ and $\mathrm{BaO}$ and examined the Type IV structure on these strained substrates. Table 2 reports results for water adsorption on two kinds of constrained surfaces: (i) the constrained clean surface with rumpling; and (ii) the bulk truncated surface without rumpling. In contrast to

Table 2 Adsorption energy of water in the Type IV structure on various $\mathrm{CaO}$ and $\mathrm{BaO}$ substrates. $a_{0}(\mathrm{CaO}$, clean $)$ and $a_{0}(\mathrm{BaO}$, clean $)$ indicate that the substrates adopt the lattice constants and fully relaxed clean surface structures of $\mathrm{CaO}$ and $\mathrm{BaO}$, respectively. $a_{0}\left(\mathrm{CaO}\right.$, bulk) and $a_{0}(\mathrm{BaO}$, bulk) indicate that the substrates have bulk truncated surface structures at the $\mathrm{CaO}$ and $\mathrm{BaO}$ lattice constant, respectively. $E_{\text {ads }}$ is defined in eqn (1), where, however, the total energies of the clean surface $\left(E_{\mathrm{MN}}\right)$ are the total energy of each fully relaxed or bulk truncated surface with the indicated lattice constants instead. In each case it is indicated in parentheses if the water is intact (Int) or dissociated (Diss). All values are in eV.

\begin{tabular}{lllll}
\hline$E_{\text {ads }}$ & $a_{0}(\mathrm{CaO}$, clean $)$ & $a_{0}(\mathrm{BaO}$, clean $)$ & $a_{0}(\mathrm{CaO}$, bulk $)$ & $a_{0}(\mathrm{BaO}$, bulk $)$ \\
\hline $\mathrm{CaO}$ & -0.61 (Int) & -1.39 (Diss) & -0.60 (Int) & -1.21 (Diss) \\
$\mathrm{BaO}$ & -0.45 (Int) & -1.11 (Diss) & -0.46 (Int) & -1.00 (Diss) \\
\hline
\end{tabular}

a regular $\mathrm{BaO}$ substrate, it is found that water does not dissociate on a $\mathrm{BaO}$ substrate which is compressed to the $\mathrm{CaO}$ lattice constant. Similarly expanding $\mathrm{CaO}$ to adopt the $\mathrm{BaO}$ lattice constant makes the perfect unrelaxed $\mathrm{CaO}$ substrate reactive enough to dissociate water. Thus, the lattice constant of the substrate is of key relevance to the question of water adsorption and dissociation.

\section{Discussion and conclusions}

Although there has been considerable interest in working out the details of water adsorption, molecular level understanding is lacking for many water adsorption systems. With a view to better understanding water adsorption on insulating inorganic substrates, we have performed a systematic trend study of water monomer adsorption and dissociation. The surfaces examined have comprised a broad range of rocksalt (001) surfaces, including alkaline earth oxides, alkaline earth sulfides, alkali fluorides and alkali chlorides. The focus has been on understanding the inherent reactivity of each substrate towards water monomers and so we have not considered other (interesting) issues such as water clustering or the role of substrate defects.

We find that water remains intact on most surfaces considered and only a few specific adsorption structures are favoured. This is generally either a structure with the water molecule above an individual metal ion with the molecular plane almost parallel to the surface (Type I) or a structure with the molecular plane along the surface normal and with the $\mathrm{OH}$ bond(s) directed at the surface (Type II or III). When water dissociates a single structure has been identified which involves an adsorbed hydrogen on a surface oxygen site and a hydroxyl above the adjacent metal ion (Type IV). The Type I adsorption structure is reasonably similar to the water monomer adsorption structure predicted by theory for water on a large number of metal surfaces. ${ }^{16}$ Analysis of the underlying electronic structures shows that the adsorption structures on the insulating substrates considered here are dictated mainly by electrostatics. This is in contrast to the water-metal adsorption systems where the preference for water monomer adsorption above individual metal atoms results from a weak covalent (orbital overlap) interaction.

The stability of the water monomer increases upon descending the oxide and fluoride series but remains rather constant upon going down the chloride and sulfide series. As the lattice constant of the substrate increases (as happens upon descending each series) the hydrogen bonding interaction with the substrate is increased because the molecule can approach the substrate more closely in the Type II (or III) adsorption structure. Conversely the water-cation interaction decreases as a series is descended (disfavouring the Type I structure) and so which monomer structure is preferred is a result of this balance between water-cation bonding and hydrogen bonding.

Water monomers only dissociate on three of the substrates considered $(\mathrm{CaO}, \mathrm{SrO}$, and $\mathrm{BaO})$. The stability of the dissociated state increases as the (oxide) series is descended and the flexibility of the substrate is shown to be a key factor in determining the stability of the dissociated state. The flexibility of the substrate is particularly important for the stability of the 
dissociated state because the substrate atoms beneath the adsorption complex are drawn out of the surface in the presence of the $\mathrm{OH}$ and $\mathrm{H}$ adsorbates. We have also found that the substrate lattice constant plays a key role in determining the relative stability of the intact and dissociated states and that varying the substrate lattice constant provides a means to tune the adsorption state. For example, in the case of $\mathrm{BaO}$ we have shown that by contracting the lattice constant from the equilibrium $\mathrm{BaO}$ value to the value of $\mathrm{CaO}$ a switch in the stable adsorption state from a dissociated water structure to an intact water structure is observed. Substrate strain has been widely used to alter the chemical reactivity of substrates, most notably metal surfaces ${ }^{59,60}$ through $e . g$. alloying. Since some of the inorganic substrates considered here are now routinely prepared as thin film epitaxial heterostructures there is the potential to control their lattice constant and hence the chemical state of water on these systems.

\section{Acknowledgements}

J.C.'s work is supported by the Royal Society through a Newton International Fellowship. A.M.'s work is supported by the European Research Council, the EURYI scheme (see: www.esf.org/euryi), and the EPSRC. Computational resources from the London Centre for Nanotechnology and UCL Research Computing are gratefully acknowledged. J.C. is grateful to Matthias Scheffler for support. Partly via our membership of the UK's HPC Materials Chemistry Consortium, which is funded by EPSRC (EP/F067496), this work made use of the facilities of HECToR.

\section{References}

1 A. Hodgson and S. Haq, Surf. Sci. Rep., 2009, 64, 381.

2 G. E. Brown Jr., V. E. Henrich, W. H. Casey, D. L. Clark, C. Eggleston, A. Felmy, D. W. Goodman, M. Grätzel, G. Maciel, M. I. McCarthy, K. H. Nealson, D. A. Sverjensky, M. F. Toney and J. M. Zachara, Chem. Rev., 1999, 99, 77.

3 M. A. Henderson, Surf. Sci. Rep., 2002, 46, 1.

4 P. Fenter and N. C. Sturchio, Prog. Surf. Sci., 2004, 77, 171.

5 A. Verdaguer, G. M. Sacha, H. Bluhm and M. Salmeron, Chem. Rev., 2006, 106, 1478.

6 G. E. Ewing, Chem. Rev., 2006, 106, 1511.

7 M. W. Kanan and D. G. Nocera, Science, 2008, 321, 1072.

8 M. W. Kanan, Y. Surendranath and D. G. Nocera, Chem. Soc. Rev., 2009, 38, 109.

9 D. Wolf, M. Barré-Chassonnery, M. Höhenberger, A. van Veen and M. Baerns, Catal. Today, 1998, 40, 174.

10 Q. Fu, H. Saltsburg and M. Flytzani-Stephanopoulos, Science, 2003, 301, 935.

11 N. Kumar, P. R. C. Kent, A. V. Bandura, J. D. Kubicki, D. J. Wesolowski, D. R. Cole and J. O. Sofo, J. Chem. Phys., 2011, 134, 044706.

12 K. D. Kreuer, Annu. Rev. Mater. Res., 2003, 33, 333.

13 J. K. Nørskov, T. Bligaard, J. Rossmeisl and C. H. Christensen, Nat. Chem., 2009, 1, 37.

14 J. K. Nørskov, T. Bligaard, B. Hvolbæk, F. Abild-Pedersen, I. Chorkendorff and C. H. Christensen, Chem. Soc. Rev., 2008, 37, 2163.

15 A. Michaelides, Z.-P. Liu, C. J. Zhang, A. Alavi, D. A. King and P. Hu, J. Am. Chem. Soc., 2003, 125, 3704.

16 A. Michaelides, Appl. Phys. A: Mater. Sci. Process., 2006, 85, 415.

17 A. Michaelides, A. Alavi and D. A. King, J. Am. Chem. Soc., 2003, 125, 2746 .
18 K. Andersson, G. Ketteler, H. Bluhm, S. Yamamoto, H. Ogasawara, L. Pettersson, M. Salmeron and A. Nilsson, J. Am. Chem. Soc., 2008, 130, 2793.

19 X. L. Hu, J. Klimeš and A. Michaelides, Phys. Chem. Chem. Phys., $2010, \mathbf{1 2}, 3953$.

20 L. Giordano, J. Goniakowski and J. Suzanne, Phys. Rev. Lett., 1998, 81, 1271; L. Giordano, J. Goniakowski and J. Suzanne, Phys. Rev. B: Condens. Matter Mater. Phys., 2000, 62, 15406.

21 L. Delle Site, A. Alavi and R. M. Lynden-Bell, J. Chem. Phys., $2000,113,3344$

22 R. M. Lynden-Bell, L. D. Site and A. Alavi, Surf. Sci., 2002, 496, L1.

23 C. Zhang and P. J. D. Lindan, J. Chem. Phys., 2003, 118, 4620.

24 P. J. D. Lindan and C. Zhang, Phys. Rev. B: Condens. Matter Mater. Phys., 2005, 72, 075439.

25 P. M. Kowalski, B. Meyer and D. Marx, Phys. Rev. B: Condens. Matter Mater. Phys., 2009, 79, 115410.

26 L.-M. Liu, C. Zhang, G. Thornton and A. Michaelides, Phys. Rev. B: Condens. Matter Mater. Phys., 2010, 82, 161415(R).

27 J. Cheng and M. Sprik, Phys. Rev. B: Condens. Matter Mater. Phys., 2010, 82, 081406(R).

28 O. Bikondoa, C. L. Pang, R. Ithnin, C. A. Muryn, H. Onishi and G. Thornton, Nat. Mater., 2006, 5, 189.

29 L. Liu, M. Krack and A. Michaelides, J. Am. Chem. Soc., 2008, 130, 8572; L. Liu, M. Krack and A. Michaelides, J. Chem. Phys., 2009, 130, 234702.

30 Y. Yang, S. Meng, L. F. Xu, E. G. Wang and S. Gao, Phys. Rev. E: Stat., Nonlinear, Soft Matter Phys., 2005, 72, 012602.

31 Y. Yang, S. Meng and E. G. Wang, Phys. Rev. B: Condens. Matter Mater. Phys., 2006, 74, 245409; Y. Yang, S. Meng and E. G. Wang, J. Phys.: Condens. Matter, 2006, 18, 10165.

32 V. E. Henrich and P. A. Cox, The Surface Science of Metal Oxides, Cambridge University Press, 1994.

33 C. Noguera, Physics and Chemistry at Oxide Surfaces, Cambridge University Press, 1996.

34 J. Goniakowski and C. Noguera, Phys. Rev. B: Condens. Matter Mater. Phys., 2009, 79, 155433.

35 A. Borodin, O. Höfft, U. Kahnert, V. Kempter, A. Poddey and P. E. Blöchl, J. Chem. Phys., 2004, 121, 9671.

36 G. Kresse and J. Hafner, Phys. Rev. B: Condens. Matter, 1993, 47, 558

37 G. Kresse and J. Furthmüller, Phys. Rev. B: Condens. Matter, 1996, 54, 11169.

38 J. P. Perdew, K. Burke and M. Ernzerhof, Phys. Rev. Lett., 1996 77, 3865; J. P. Perdew, K. Burke and M. Ernzerhof, Phys. Rev. Lett., 1997, 78, 1396.

39 P. E. Blöchl, Phys. Rev. B: Condens. Matter, 1994, 50, 17953.

40 G. Kresse and D. Joubert, Phys. Rev. B: Condens. Matter Mater. Phys., 1999, 59, 1758.

41 The particular PAW potentials used in our calculations include the following number of valence electrons in the potentials: $8(\mathrm{Mg})$ $10(\mathrm{Ca}), 10(\mathrm{Sr}), 10(\mathrm{Ba}), 6(\mathrm{O}), 6(\mathrm{~S}), 7(\mathrm{~F}), 7(\mathrm{Cl}), 3(\mathrm{Li}), 7(\mathrm{Na})$, $9(\mathrm{~K}), 9(\mathrm{Rb})$, and $9(\mathrm{Cs})$.

42 For example, the adsorption energy in the most stable adsorption structure is $-0.48(-0.48),-0.90(-0.91),-1.26(-1.27),-1.49$ $(-1.48) \mathrm{eV}$ with three (eight) layer slabs on $\mathrm{MgO}, \mathrm{CaO}, \mathrm{SrO}$, and $\mathrm{BaO}$, respectively.

43 H. J. Monkhorst and J. D. Pack, Phys. Rev. B: Solid State, 1976, 13, 5188

44 J. Neugebauer and M. Scheffler, Phys. Rev. B: Condens. Matter, 1992, 46, 16067

45 The PBE (experimental) values of $a_{0}$ are 4.25 (4.21) $\AA$ for $\mathrm{MgO}$, 4.84 (4.81) $\AA$ for $\mathrm{CaO}, 5.20$ (5.16) $\AA$ for $\mathrm{SrO}, 5.61$ (5.52) $\AA$ for $\mathrm{BaO}$, $5.23(5.20) \AA$ for $\mathrm{MgS}, 5.72(5.69) \AA$ for $\mathrm{CaS}, 6.06(6.02) \AA$ for $\mathrm{SrS}$, 6.46 (6.39) $\AA$ for BaS, 4.08 (4.02) $\AA$ for LiF, 4.70 (4.62) $\AA$ for $\mathrm{NaF}$, $5.42(5.35) \AA$ for KF, 5.74 (5.64) $\AA$ for RbF, $6.12(6.01) \AA$ for $\mathrm{CsF}$, $5.16(5.13) \AA$ for $\mathrm{LiCl}, 5.70$ (5.64) $\AA$ for $\mathrm{NaCl}, 6.39$ (6.29) $\AA$ for $\mathrm{KCl}$, and 6.71 (6.58) $\AA$ for $\mathrm{RbCl}$, respectively. Experimental values are taken from N. W. Ashcroft and N. D. Mermin, Solid State Physics, Thomson Learning, USA, 1976.

46 J. Carrasco, F. Illas and N. Lopez, Phys. Rev. Lett., 2008, 100, 016101 .

47 P. Broqvist, H. Grönbeck and I. Panas, Surf. Sci., 2004, 554, 262.

48 N. V. Skorodumova, K. Hermansson and B. Johansson, Phys. Rev. B: Condens. Matter Mater. Phys., 2005, 72, 125414.

49 M. Dion, H. Rydberg, E. Schröder, D. C. Langreth and B. I. Lundqvist, Phys. Rev. Lett., 2004, 92, 246401. 
50 The difference between the original vdW-DFT of Dion et al. and optB88-vdW is simply in the choice of exchange functionals, with the optB88 exchange functional yielding more accurate interaction energies than the original choice of revPBE. More details can be found in J. Klimeš, D. R. Bowler and A. Michaelides, J. Phys.: Condens. Matter, 2010, 22, 022201; J. Klimeš, D. R. Bowler and A. Michaelides, J. Phys.: Condens. Matter, 2011, 83, 195131.

51 The adsorption structures considered include ones with the oxygen of the (intact and dissociated) water located on the surface cation and anion sites as well as ones with (intact and dissociated) water bridging between these sites. At each site a variety of orientations for the molecule was examined.

52 N. H. de Leeuw and J. A. Purton, Phys. Rev. B: Condens. Matter Mater. Phys., 2001, 63, 195417.

53 B. Li, A. Michaelides and M. Scheffler, Surf. Sci., 2008, 602, L135.

54 J. Carrasco, B. Santra, J. Klimeš and A. Michaelides, Phys. Rev. Lett., 2011, 106, 026101.
55 The Type III structure in Fig. 1 is another adsorption structure that involves hydrogen bonding with the surface. The same trends and conclusions can be drawn from the Type II and Type III structures in our calculations.

$56 \mathrm{X}$. L. Hu, First principles studies of water and ice on oxide surfaces, PhD thesis, UCL, 2010, Available at www.chem.ucl.ac.uk/ice.

57 Decompositions of the adsorption energies have been performed on all surfaces. For clarity in Fig. 4, the results of the fluoride and chloride series are not shown.

58 In agreement with previous DFT studies, ${ }^{47,48}$ on the clean $\mathrm{MgO}(001)$ surface the metal ions sit inside the oxygen ions, with a negative value of the rumpling $\Delta d$. On $\mathrm{CaO}(001)$ the rumpling is almost zero and on $\mathrm{SrO}(001)$ and $\mathrm{BaO}(001)$ a positive rumpling is obtained, with the metal ions outside the oxygens.

59 M. Mavrikakis, B. Hammer and J. K. Nørskov, Phys. Rev. Lett., 1998, 81, 2819.

60 M. Gsell, P. Jakob and D. Menzel, Science, 1998, 280, 717. 